\title{
Nursing praxis, compassionate caring and interpersonal relations: An observational study
}

\author{
Margaret Fry, RN, NP, PhD ${ }^{a, *}$ \\ Casimir MacGregor, BA(Hons), MA, DPH, PhD ${ }^{\mathrm{a}}$ \\ Kate Ruperto, $\mathrm{MN}^{\mathrm{b}}$ \\ Kate Jarrett, RN, BN, MEmergNursing ${ }^{c}$ \\ Janet Wheeler, PhD Candidate ${ }^{d}$ \\ Jacqueline Fong, RN, BN, $\mathrm{MN}^{\mathrm{C}}$ \\ Wendy Fetchet, RN, GCertEmergNursing ${ }^{e}$
}

\author{
a Faculty of Health University of Technology, Sydney, Australia \\ ${ }^{\mathrm{b}}$ Emergency Services ISLHD, Australia \\ c Emergency Department St George Hospital SESLHD, Australia \\ d Liverpool Hospital WSLHD, Australia \\ e Emergency Department Shoalhaven Memorial District Hospital, ISLHD, Australia
}

Received 21 November 2012; received in revised form 20 December 2012; accepted 17 February 2013

\author{
KEYWORDS \\ Compassionate care; \\ Empathy; \\ Emergency nursing; \\ Emotions; \\ Interpersonal \\ relations; \\ Interprofessional \\ relations
}

\begin{abstract}
Summary
Background: The Clinical Initiative Nurse $(\mathrm{CIN})$ is a role that requires experienced emergency nurses to assess, initiate diagnostic tests, treat and manage a range of patient conditions. The CIN role is focused on the waiting room and to 'communicate the wait', initiate diagnostics or treatment and follow-up for waiting room patients. We aim to explore what emergency nurses' do in their extended practice role in observable everyday life in the emergency department (ED). The paper argues that compassionate caring is a core nursing skill that supports CIN interpersonal relations, despite the role's highly clinical nature.

Method: Sixteen non-participant observations were undertaken in three EDs in New South Wales, Australia. Nurses were eligible for inclusion if they had two years of emergency experience and had worked in the CIN role for more than one year. All CIN's that were observed were highly experienced with a minimum three year ED experience.
\end{abstract}

* Corresponding author at: Faculty of Health, University of Technology, Sydney, P.O. Box 123, Broadway, NSW 2007, Australia. Tel.: +61 29514 4826; fax: +61 295144835 .

E-mail addresses: margaret.fry@uts.edu.au, margaretfry1@gmail.com (M. Fry), casimir.macgregor@uts.edu.au (C. MacGregor), kate.ruperto@sesiahs.health.nsw.gov.au (K. Ruperto), kate.jarrett@sesiahs.health.nsw.gov.au (K. Jarrett), janet.wheeler@uts.edu.au (J. Wheeler), Jacqui.Fong@sesiahs.health.nsw.gov.au (J. Fong), wendy.fetchet@sesiahs.health.nsw.gov.au (W. Fetchet).

1574-6267/\$ - see front matter @ 2013 College of Emergency Nursing Australasia Ltd. Published by Elsevier Ltd. All rights reserved. http://dx.doi.org/10.1016/j.aenj.2013.02.003 
Results: The CIN observations revealed how compassionate caring was utilised by CIN's to quickly build a therapeutic relationship with patients and colleagues, and helped to facilitate core communication and interpersonal skills. While the CIN role was viewed as extended practice, the role relied heavily on compassionate care to support interpersonal relationships and to actualise extended practice care.

Conclusion: The study supports the contribution made by emergency nurses and demonstrates how compassionate caring is central to nursing praxis. This paper also demonstrates that the CIN role utilises a complex mix between advanced clinical skills and compassion that supports interpersonal and therapeutic relationships. Further research is needed to understand how compassionate care can be optimised within nursing praxis and the duty of care between nurses and patients, nurses and other health care professionals so that future healthcare goals can be realised.

(c) 2013 College of Emergency Nursing Australasia Ltd. Published by Elsevier Ltd. All rights reserved.

\section{What is known}

- Nursing was founded on being compassionate and caring for those suffering. However with the increasingly complex clinical and technological nature of modern health care nursing is now more complex and multi-dimensional.

- Nursing best practice is about ensuring a calm safe environment, a positive friendly culture, well managed care with efficient delivery, patient centred care; and finally good team working and relationships.

\section{What this paper adds}

- Compassionate care is a central aspect of nursing praxis even for expert nurses undertaking extended practice roles.

- Health care organisational pressures can impact on a nurse's ability to engage in compassionate care.

- A culture of care centred the nurses' ability give compassionate care and good communication interpersonal relations and team work is important for nurses and doctors in their duty of care.

\section{Introduction}

In this paper we explore the nursing praxis of the Clinical Initiative Nurse (CIN) in the emergency department (ED). Specifically we examine the role of the expert nurse as a compassionate carer through their interpersonal relations with patients and colleagues. The CIN role was first implemented in 2002 in New South Wales (NSW), Australia. ${ }^{1}$ The ED role required experienced nurses to operate within an extended scope of practice in acute clinical areas, to assess, initiate diagnostic tests and treat and manage a range of patient conditions. Aspects of the CIN role have similarities with roles, such as the Clinical Nurse Specialist (CNS) in the USA, Emergency Nurse Practitioner (ENP) in Britain or the Australian Clinical Nurse Consultant (CNC) and the rapid assessment nurse, fast track nurse, stat nurse and advanced practice nurse roles seen in other Australian states. $^{2,3}$
In 2010 the NSW Ministry of Health redefined the CIN role with the focus on caring for waiting room patients. ${ }^{1}$ The role of the CIN was now to 'communicate the wait' and prioritise the care of patients in ED waiting rooms, initiate diagnostics and/or treatment follow-up especially pain management. The goal of the CIN role was to achieve timely and appropriate patient care and with other ED team members to provide co-ordinated care. ${ }^{4-6}$

The high paced, complex nature of the ED means that nurses' work closely with their patients and colleagues in a context that involves high emotions and tensions. The practice of compassionate caring in nursing praxis entails negotiating the relationship between physical and emotional well-being. Caring is arguably the defining characteristic of nursing. Caring has been defined as the mental, emotional and physical effort involved in looking after, responding to and supporting others'. ${ }^{7}$ Compassion involves the 'deliberate participation in another person's suffering, not merely [the] identification of the suffering, but identification with it'. ${ }^{8}$ The highly specialised nature of the CIN role is its most commonly noted feature. We argue that despite the clinical expectations of this expert nurse undertaking extended practice activities, compassionate caring is central to their nursing praxis.

This paper forms part of a research project that examined the role of an expert nurse undertaking extended practice. The aim of this paper is not to examine the perceptions of the CIN role, as this has been explored elsewhere, ${ }^{9}$ but rather to examine what the CIN does - the praxis of being a CIN in observable everyday life. By using non-participant observation we were are able to examine what the CIN does and how they interact with their patients and peers.

\section{Methods}

This was a multicentre study and formed part of a larger qualitative exploratory study of the CIN role.

\section{Study sites}

The study was undertaken across three EDs in the state of New South Wales, Australia and included a metropolitan university tertiary referral hospital, a regional referral hospital and a regional hospital to maximise findings (Table 1). ${ }^{10,11}$ 
Table 1 Hospital study site demographics.

\begin{tabular}{|c|c|c|c|}
\hline & Hosp. $1^{a}$ & Hosp. $2^{b}$ & Hosp. $3^{c}$ \\
\hline Bed numbers & 550 & 581 & 213 \\
\hline Presentation rate & 59,700 & 53,000 & 34,500 \\
\hline Admission rate & $25,074(42 \%)$ & $23,320(44 \%)$ & $11,079(32 \%)$ \\
\hline
\end{tabular}

\section{Sample and recruitment}

A purposeful sample of emergency nurses who undertake the CIN role was sought. A total of sixteen in depth non-participant observations were undertaken through the use of the ethnographic method. All CIN's observed were highly experienced with a minimum three year ED experience, with the majority ranging from 5 to 10 years ED experience. Non-participant observations (four-five hours) were conducted to develop an understanding of how CIN's make sense of their practice. Observational data was collected and analysed using thematic analysis based on ethnographic principles.

\section{Data analysis}

All data were de-identified and codes identifying participants were stored as separate electronic files. Thematic analysis was conducted using inductive reasoning guided by Gibbs's ${ }^{12}$ framework which included: (1) transcription, familiarisation and immersion into data; (2) code building; (3) dis/confirmatory theme development; and (4) data consolidation and interpretation. The investigators discussed and reviewed the emerging coding and organisation of themes for this paper. Based upon Gibbs's ${ }^{12}$ framework six main themes emerged from the data. These themes provided a systematic approach for interpretation. ${ }^{13,14}$ We have used fieldnotes to demonstrate, how the expert nurse engaged in their daily practice of compassionate care and to create meaningful units of analysis.

\section{Ethics approval}

Ethical approval was obtained from the local Human Research Ethics Committee and conformed to the 'National Statement on Ethical Conduct in Research Involving Humans' by the National Health and Medical Research Council of Australia. The ethical conduct of research was maintained during and after the research. Consent was obtained from all participants and every effort was taken to respect participant participation without compromising health and safety as outlined in the Code of Ethics for Nurses in Australia. All data and identifiable hospital and participant features were coded to maintain confidentiality, trust, and privacy and stored in password protected files.

\section{Results}

Sixteen observations (over $66 \mathrm{~h}$ ) were undertaken with thirteen females and three males. Six themes emerged that centred on compassionate caring and interpersonal relations: positive body language and emotions in nursing praxis; CIN calming strategies for patients; negative body language; CIN's professional and personal conflict; the ED doctor and the CIN; and nursing mentoring and teamwork - the CIN and the assistant in nursing or AIN.

What we see from the observations is how these expert nurses drew on their experience of compassionate caring to quickly build a therapeutic relationship with patients, by utilising core communication and interpersonal skills. By using these compassionate caring skills the CIN's were able to quickly establish a relationship with the patients' that enabled them to execute their clinical skills. Despite the clinical nature and urgency of the expert nurses advanced practice activities, the role deeply relied on core nursing skills of communication and interpersonal relations to actualise the practice of compassionate caring.

\section{Positive body language and emotions in nursing praxis}

The first theme to emerge from the observations of CIN practice was the strong interpersonal nature of the position. Despite the CIN position being seen as extended practice or arguably an advanced practice role, the core tasks of the CIN are similar to any other nursing position. The core skills of nursing are strong interpersonal connections to patients and colleagues. Many of the observations demonstrated that the CIN's practice was closely related to their ability to interpersonally relate to patients and engage in compassionate caring - especially through the use of eye contact, empathy, humour, small talk and touch. As one CIN noted:

First being decent to people makes a difference it's so undervalued this aspect (013).

Direct positive body language, especially eye contact was one of the most prominent attributes of positive body language:

CIN has a quiet voice - goes down to patient level - eye contact: unable to hear - patient feels unwell (010).

CIN calls female in by first name (knows patient from previous visit). Patient rambling a bit with story/history but giving details eventually. (CIN giving eye contact/caring/empathy) - checks blood pressure/pulse on automatic monitor (01).

Other acts of positive body language, such as the use of touch were also important. Touch in these circumstances 
was a way to add comfort to the patent in times of stress and uncertainty:

CIN walks through to waiting room and talks to elderly man - touches him on the knee, 'you can eat and drink while [you are] waiting' joking with patient 'high protein drink, yoghurt to eat?' (04).

But it was not only the act of touching that was reassuring, but the offer of comfort in these times of distress:

CIN goes into CIN bedroom - asks Doctor if she is ok. - CIN gets charts 'are you allergic to anything? Relative answers 'yes'. To patient: I know it's not a nice bit is it darling. Do you want to hold my hand?' CIN holds patient's hand as arterial blood gas obtained by Doctor (015).

However, in the following observation we can see that the situational problems of the emergency department could also make positive body language difficult:

Eye contact between CIN and patients occurred, but CIN was often distracted by other staff coming into the room and asking questions/discussing other patients. The CIN did not sit or lower herself down to the level of the patient - was always standing. Empathic interaction by CIN with patients - often difficult due to constant interruptions and workload, and apparent interpersonal skills not fully developed (012).

We can see from these observations that compassionate caring through the use of positive body language is used to establish a therapeutic relationship between the expert nurse and patient. We can also observe from the experienced CIN's praxis that these forms of compassionate caring executed through communication and interpersonal relations became unreflective or a habitual action. Compassionate caring can also be seen in the next theme to emerge from our data of the calming strategies used by the CIN on patients.

\section{CIN calming strategies for patients}

Positive body language was an important part of CIN's compassionate caring with patients. However, there were other aspects of compassionate caring employed by the CIN that were used in-conjunction with the positive body language that had a calming effect for the patient, to help them cope with their stress and uncertainty. One such strategy of the CIN was the use of conversation, chit chat or small talk during patient interactions. In the following observations we can see how the CIN's use of conversation was a way to alleviate the patients stress of an impending procedure:

CIN listens to and asks questions and history - behaviour of previous presentations for ED. Goes into triage room for IV equipment - 'has been so busy this morning' (to patient). Patient stays on blood monitor. CIN changes to right arm of patient, has a wound from a chicken landing on his arm. CIN - 'don't want infection travelling up your arm'. CIN prepares IV site - comments on bruise on right hand. Discusses chooks and farming things, eggs free range. IV cannula inserted - blood samples taken. Talking about animals to wife/women - 'they are beautiful'. Secures IV cannula (010).
Even in tense situations when the patient wasn't responsive to the CIN, the nurse attempted to have a calming effect on the patient and those around them:

Paediatric Registrar asked if CIN would help to take bloods from a young girl (2 year old). CIN goes behind curtain. Girl asleep - being quiet not to be disturbed, as she was distressed before. CIN prepares equipment \& holds girl's arm while Paediatric Registrar cannulates and obtains blood. Mother holds girl (cuddles) on bed. CIN not saying anything - Girl screaming continuously. After procedure - CIN 'you were a good girl' - quietly/kindly (01).

The CIN's ability to engage in compassionate caring in their interpersonal relationships with patients was demonstrated through positive body language strategies which assisted to calm patients during stressful or challenging events. However, the backdrop of conflicting pressures made their role in supporting and enabling compassionate care at times challenging. This was because when the CIN was under stress and experiencing challenging circumstances, positive body language and emotions were impacted.

\section{Negative body language}

During the observations when the CIN experienced stress or pressure, such as when the Waiting Room was busy or full, these contextual factors would impact on the CIN's body language and how they inter-related with patients:

CIN very, very busy - trying to keep on top of workload. Not smiling looks stressed. Moving from patient to patient notes in computer. Walks to triage room-picks up patient notes - call patient from waiting room - walks up CIN (laughs) 'ok come with me' (013).

As observation 13 demonstrated, despite the stressful situations that the CIN encountered they do not lose their professionalism. In another observation we can also see how stressful situations can impact on a CIN's interpersonal relations.

This CIN looked tense and walked very quickly to wherever he was going. His facial expression was serious but the words he used were similar to the other CIN observations. He did not seem as relaxed or to be able to relate as well to the patients in the waiting room as the other CIN's. He stood upright while talking to patients in waiting room. Did not attempt to sit - converse at the patients at eye level (03).

This change in the CIN's body language to a more negative body language has important implications for the practice of compassionate caring, as CINs are not able to connect with the patients. This can be seen in Observation 15:

The CIN describes difficulty not being able to 'eyeball' patients in waiting room and patients not able to 'eyeball' CIN. CIN can't monitor patients, and patients feel as though they are not getting care - leading to distress.

These observations highlight how stress and other factors can impact on the CIN's compassionate caring and their interpersonal relations. When a CIN was looking stressed 
and under pressure this often resulted in negative body language. The negative body language impacted on the CIN's ability to provide compassionate care as it meant that they found it difficult to connect at an interpersonal level with patients. But also this negative body language could be interpreted by patients that they are not getting good care.

\section{CIN's professional and personal conflict}

Another theme that emerged was that the CIN's ability to provide compassionate care was also restrained by organisational pressures which brought about a tension between professional and personal conflicts. As an expert nurse undertaking advanced practice, the CIN often found that their role was to strictly adhere to professional duties and protocols. But these duties and protocols often challenged the CIN's personal emotions and sensibilities. We can observe this in the following observation:

The CIN advises patient that she is to go into the waiting room and wait for the doctor to see her. Patient: 'I've been waiting since 8 o'clock to see them'. CIN: 'I believe you'. CIN looks to see wait time - there are seven patients in-front of you - it's a bit long to wait. We are trying to do our best. Do you want a blanket to wrap around you? (patient complains that they feel cold). Temperature normal - patient gets off bed and puts shoes on. CIN: 'I'll walk out with you'. You can't stay here as we need this space. (CIN turns to observer) this is when I want to cry. She (patient) won't move, but she can't stay here - there are more patients to be seen (012).

However, this juggling act between the expert nurse's professional responsibilities and their personal emotions and feelings was not only towards patients but also other staff:

2:1:1 gives update of other patient - describing placement of patients. CIN explains 'patient shuffling' discussing how In-charge nurse may ask where are the patients, why she hasn't done obs on some patients. Why are the patients waiting so long in the waiting room. CIN stressed/annoyed. CIN sees triage nurse-advises, she is in angry with her boss - 'I always say sorry' but I should say something back like are you kidding - I've got 12 patients out there [in the waiting room] (013).

This constant tension between professional and personal boundaries is deeply tied to the CIN's commitment, resolve and responsibilities that they share in the ED. In the following observation we see that the CIN personally feels that $\mathrm{s} /$ he must set boundaries with other colleagues about how they can help.

In CIN role you often have Doctors/ RN's asking you to do things and sometimes you have to say NO because it's too much. 'You have to prioritise'. CIN stops documentation and turns to patient with nebulizer and says 'you're right [patient name]?' Patient nods 'yes'...CIN suddenly goes very pale and has to sit down 'I'm going to faint'. Has to sit down + feels faint. Doctor sitting next to her and fast track nurse comes in - advises CIN to have some lunch. Fast track nurse: 'You've not had anything [food/drink] all day (015).
This demonstrated that the fulfilment of CIN role was deeply tied to the CIN's own personal commitment, which often meant that they stretched their bodies and self to their own personal and physical limits.

\section{The ED doctor and the CIN}

In this section we see another aspect of the interpersonal challenges the CIN faces in their nursing praxis. As an expert nurse undertaking advanced activities the CIN was not there to challenge the Doctor or Nurse Practitioner or take over their duties, but to help facilitate patient care. But, in some instances the CIN took over some of the clinical practice from the Doctor, as we can see in observation 1:

CIN goes to teenager who was vomiting \& had headache - CIN tidying up after Doctor tried to insert IV cannula. CIN inserted IV for Doctor, as Doctor had tried twice before. CIN starts IV fluid infusion pump. Goes and talks to Doctor - re adjusts rate.

There were also instances when CIN tasks or activities were unclear. In observation 1, we saw that the Doctor was having trouble inserting the cannula, so the CIN steps in to help. But often the CINs find themselves in a position where they are often pre-empting what the ED Doctor many need in order to improve treatment efficiency:

A Doctor comes to CIN to ask to call a patient into fast track, he needs dexamethasone dose and IV cannula. Doctor assumes CIN will do. CIN tells Doctor to put the cannula. CIN: 'I don't want to go back too soon as I want the Doctor to get the cannula in'. Doctor having trouble putting in cannula. CIN helps Doctor with insertion of cannula. Doctor gets cannula in and gives drug. Takes blood, CIN puts dexamethasone infusion on. Checks patients pulse and gets blood pressure machine, checks vital signs. The Doctor just wants you to do their bloods and hand it over. But you do it to keep things moving. You know the Doctor's going to ask me to do it anyway. Imagine they [the patient] have been [waiting] out there for three (3) hours for the doctor and then wait a half hour for bloods. He didn't need it, but I thought he was going to be sicker than I thought. I was relieved when I saw him. He is not too bad. CIN goes to order bloods at the printer $(011)$.

We can also see from the observations that the Doctor and the CIN work as a team, often bouncing ideas of each other, and helping out with various jobs. The main goal was always the treatment of the patient. As can be seen in the following observation where the Doctor is helping the CIN:

CIN advising patient they will take bloods, Doctor arrives - CIN explains swollen right knee, very rigid (all over) hard to get moving, wife giving history to Doctor. Doctor helping CIN with bloods and cannula - patient a bit confused/bending arm (or trying to) - Doctor took syringe with blood in away (20mls syringe full) - he will do pathology. CIN, entering presentation of patient to ED [computer] \& filled in pathology page \& gave printed forms to Doctor (03). 
However, in stressful and complex situations tension can surface within any team. In the following observation we can see the implications of an error made during a patients care. But rather than the error creating tension within the inter-professional relations their focus was on their duty of care to the patient, rather that any personal or professional rivalries:

CIN trying to organise adequate pain relief for dying patient-advising nursing home that CIN is allowed/not allowed to do regarding pain relief/analgesia - discussing medication prescribed by ED Doctor. Double checking analgesia with Doctor in ED. Doctor wrote prescription (had made error in script) - said he was not annoyed with CIN for highlighting he had made mistake (acknowledged) too large dose of schedule 8 drug $^{1}$ (05).

The CIN is an enabler for ED treatment their job is to make the treatment happen. The relationship between the Doctor and the CIN is centred on teamwork, and the duty of care towards the patient. These observations suggest that the CIN role is there to facilitate emergency care by promoting, enabling and sharing dialogue between the ED doctor and the CIN and the wider treatment team. This teamwork approach suggests that CIN's nursing praxis was deeply tied to interpersonal relations not only with patients, but also colleagues in facilitating patient centred care. A similar pattern of interpersonal relations can also be seen in the CIN's interactions with other nurses.

\section{Nursing mentoring and teamwork: the $\mathrm{CIN}$ and the AIN}

The CIN's interactions with other nurses, especially the enrolled nurse (EN) and assistant in nursing (AIN) ${ }^{2}$ detailed another kind of working interpersonal relationship. The CIN's relationship with these other nurses was also centred on team work, but involved a mentoring role by the CIN to facilitate the learning of compassionate care:

Observation 14:

Doctor opens, AIN arrives and CIN smiles 'Oh here you are' (relief in voice) CIN gives AIN (3rd year nursing student) update of patients. CIN has gentle voice, even tone, expresses concern regarding patients condition I haven't even touched some patients (re care)' to AIN. 'Talking about 3 o'clock we need to start doing obs' of patients in and out of waiting room. AIN goes to waiting room - CIN - 'put your beautiful smile on because she [patient] may be quiet and having to wait for so long. AIN smiles 'ok'. CIN looks at female patient as she walks with AIN into CIN bedroom 'oh she seems quite nice'.

The CIN asserts to 'put your beautiful smile on' this could be seen as a form of professional socialisation to help other

\footnotetext{
1 Schedule 8 drugs in Australia refer to drugs that are categorised as addictive such as morphine, opium and many others.

2 Enrolled nurse (EN) and assistant in nursing (AIN) are nursing support roles that only required a certificate or diploma level qualification. Their main duties are to provide physical and emotional support to the registered nurse (RN) and are often under their supervision.
}

nurses learn the practice of compassionate care, but also how to be a compassionate nurse. The team work approach of helping each other became important in periods of stress and high work load. In observation 14 we can see the importance of this teamwork:

CIN to AIN: 'you do your obs. I will review'. AIN advises patient needs repeat ECG in CIN bed - CIN sighs (frustration) 'ok'. CIN-AIN talk in corridor: IV reviews, observations AIN has done. 'Thank god we've got her [AIN] to help me' - smiles at AIN. Looking at waiting room list and entering AIN observations, she has done on a patients.

The teamwork was not only facilitating patient care, but is also carried over to facilitate between health care professionals. In another observation the AIN is working with a CIN in a teamwork scenario, but this time is liaising with other health care professionals

AIN advises CIN that doctors say patient cannot return to the waiting room - CIN asks AIN if she can advise 2:1:1, AIN placing armband on patient and reviewing obs machine. CIN in corridor discussing care with AIN recording what the patient's vitals are and when to repeat them (016).

These observations demonstrate how interpersonal relations, especially teamwork in CIN nursing praxis focused on teaching less experienced nurses the practice of compassionate caring - how to be a nurse. By teaching EN's and AIN's how to be a nurse in their interpersonal relations with other nurses, compassionate caring can be thought of as a core nursing skill and a central part of professional socialisation. Being a nurse means utilising compassionate caring and interpersonal relations as a foundation for communication and as a core aspect of their nursing praxis and professionalisation.

\section{Discussion}

The study has provided a rich understanding of the role of an expert nurse conducting extended practice roles and the use of compassionate caring as a central part of their everyday nursing praxis and interpersonal relationships. The findings support the valuable and principled contribution of the compassionate caring of nurses in managing patients and affirmed how the role of an expert nurse undertaking advanced activities relies heavily on core nursing skills (i.e. compassion) to build a therapeutic relationship with patients, and together with the help of doctors and fellow nurses through teamwork fulfil a duty of care towards the patient.

Davidson and Williams ${ }^{15}$ in their research into factors that influence compassionate care in clinical practice have described how 'technical functions are more likely to be carried out by more senior or experienced nurses drawing them away from direct nursing care'. However, our observations suggest that for the expert nurses compassionate caring can co-exist with clinical practices and remain a core aspect of their nursing praxis. Our study also highlights how compassionate caring through the use of eye contact, smiling and touch are important aspects of nursing praxis. 
Caris-Verhallen et al. ${ }^{16}$ have shown how non-communicative action, such as eye contact, affirmative head nodding, smiling, body position and touch are central to nurse-patient interpersonal relations.

Our observations show how compassionate caring is central to CIN nursing praxis. This is in spite of the fact that these compassionate caring skills or their value are not often recognised by the organisation or certain sections of society. ${ }^{17}$ Phillips ${ }^{18}$ suggests that the problem of the recognition of emotions in nursing is that it would diminish the professional standing of the profession. However, Phillips ${ }^{18}$ comments are situated against the context of the technical, task-oriented nursing grounded in biomedicine that is seen as more 'real' than compassionate nursing. However, what our data suggests is that it is hard to separate the compassionate or emotional aspects of nursing from extended/advanced practice clinical skills. Expert nurses (in this instance the $\mathrm{CIN}$ ) use their compassionate caring in interpersonal relations, as another skill set that complements their advanced clinical skills, in order to care for their patients and work in the ED team.

Our observations have also highlighted how the core interpersonal skills of the CIN, such as positive body language, touch etc. enabled nursing praxis to be directed towards compassionate caring. Despite the high clinical demands of the CIN role, the essential elements of the role were centred on compassionate caring through interpersonal relations which had become an embodied skill of nursing praxis. By skill we are not suggesting simply that compassionate caring is merely the 'embodiment of knowledge', but rather it became an embodied combination of physical, perceptual and behavioural change of the individual subject so that $\mathrm{s} /$ he could accomplish tasks that prior to enskillment were impossible. ${ }^{19}$ The practice of compassionate caring was not something one could brandish about, but rather something that one does.

Our observations also describe how organisational barriers influenced the capacity of nurses to practice compassionate nursing. As the CIN role is involved in 'managing the wait' they are in direct contact with the public, and faced with managing the mismatch between policy rhetoric and practical reality. ${ }^{20}$ This background of conflicting pressures made the CIN role as a compassionate carer even more challenging. In our examination of the CIN's professional and personal conflict, we can observe a tension in the CIN's praxis between what was expected of the CIN through protocols etc. and how the CIN personally felt or expressed their emotions in relation to their context. Thus when there is a gap between what we feel and what we ought to feel, we engage in what Staden ${ }^{17}$ has termed emotional management strategies. The first of these strategies is called surface acting in which we change our inner feelings to correspond to how we might appear. ${ }^{17}$ The second strategy is deep acting, whereby we change our inner feelings using a variety of methods, so that feelings we project are the feelings we want to feel. ${ }^{17}$ We can see from our data that CINs engage in the emotional management strategy of surface acting, but rather than changing their inner feelings, the CIN changes their praxis. As we saw in observation 13, the CIN disagreed about certain hospital protocols, and her inner most feelings also disagreed, but her praxis was to fulfil the protocols in contradiction to her feelings.
The emotional management between the disjuncture of how people feel and what they do is an important theme for nursing praxis, and suggests and that more research needs to be done in order to understand this emotional complexity within expert nursing roles.

The study demonstrated that CINs work closely with Doctors and mentor less experienced nurses, which facilitates professional socialisation and an understanding of a duty of care towards patients. The CIN is in a unique position to contribute to emergency care culture because of their highly developed clinical skills and use of compassionate caring in nursing praxis. It is the connection between these two skills sets (clinical and compassionate care) that can create optimum care for patients. In a recent study conducted in an ICU it was found that nurse-doctor collaboration had been shown to positively affect patient outcomes. ${ }^{21}$ Aitken and others ${ }^{22}$ have also illustrated where effort is concentrated on creating good team work and environment for staff, improvements in patient care can result. These studies outline the importance of interpersonal relations in health care. Moreover compassionate care should not so much be seen as a complement to clinical skills, but should be the central starting point of promoting best practice. As West and others ${ }^{23}$ have stated 'cultures of engagement, positivity, caring, compassion and respect for all-staff, patients and the public - provide the ideal environment within which to care for the health of the nation'. It is therefore critical that nursing research continues to record and contextualise the positive collaboration between nurses and their colleagues, like that observed in our study. It is also critical that hospitals promote an organisational culture of care that centres on compassionate caring, good team work, and good interpersonal relations.

\section{Limitations}

Several limitations to the study need to be acknowledged. Two such limitations that we did not examine include the impact that gender could have had on CIN interpersonal interactions. We also did not examine patient experiences of the CIN's interpersonal relations in the ED. While other researchers may have chosen to present different information, the findings of this study would remain familiar to those who undertake the CIN role. In fact the implications of gender and patient experiences of CIN practices of care could be useful departure points for future research that could enhance our discussion of CIN's interpersonal relations.

Another limitation was the study's focus on experienced CINs who may have different views and practices when compared to those with less experience. Nonetheless, experienced CINs were sought because they would be confident in their skills and role compared to a less experienced CIN. Despite this concern the sample group was representative but not inclusive of all CINs throughout NSW. The context of care was similar to most EDs nationally and internationally. In addition, this study was conducted primarily in mixed adult and children hospitals, so little inference can be extrapolated to CIN roles in dedicated children's hospitals. However, the multicentre study assisted to strengthen findings and applicability. 


\section{Conclusion}

This paper has provided a deeper understanding into the CIN role and how compassionate caring and its interpersonal factors are central to nursing praxis. The study supports the contribution made by emergency nurses and demonstrates that nursing entails a complex mix between clinical skills and good interpersonal relationships in compassionate caring. The findings of this study have important implications for how we understand the role of the expert nurse undertaking extended practice roles.

Given the importance that compassionate caring has to interpersonal relations, collaboration and nursing praxis more research needs to be done to understand how emotions, communication and interpersonal relations can be optimised within nursing praxis and the duty of care between nurses and patients, nurses and nurses, and nurses and doctors. By recognising the importance of compassionate caring as a critical skill in nursing, a more appropriate culture of care can be achieved.

\section{Provenance and conflict of interest}

There is no conflict of interest. This paper was not commissioned.

\section{Funding source}

This study was funded by a NSW Health Nursing and Midwifery Office Innovation Scholarship. We wish to acknowledge New South Wales Ministry of Health Nurses and Midwives Office for providing an innovation scholarship.

\section{Statement of authorship}

MF, KJ and KR conceived and designed the study. MF secured funding. MF, KJ, KR, and WF developed the study protocol. MF, JF, KR and JW supervised and collected the data. MF, CM, $\mathrm{KJ}, \mathrm{JW}$ and KR and ED analysed the data. MF, CM, KJ, WF, JW, JF and KR prepared and approved the manuscript.

\section{Acknowledgments}

We wish to acknowledge New South Wales Ministry of Health Nurses and Midwives Office for providing an innovation scholarship.

\section{References}

1. Department of Health and Ageing: Expert Panel - Review of Elective Surgery and Emergency Access Targets under the National Partnership Agreement on Improving Public Hospital Services: Supplementary Annexure: New South Wales - Clinical
Initiative Nurses in Emergency Departments. Canberra: Australian Government; 2011.

2. Hudson P, Marshall A. Extending the nursing role in emergency departments: challenges for Australia. Aust Emerg Nurs J 2008;11:39-48.

3. Carruthers J, Sykes D, Blackmore D. Work analysis project: emergency department, intensive care and radiology departments. In: Services BPaDoH, editor. Melbourne: Bearing Point and Department of Human Services; 2004.

4. Fry $M$, Jones $K$. The clinical initiative nurse: extending the role of the emergency nurse, who benefits? Aust Emerg Nurs J 2005; 8:9-12.

5. Fry M, Borg A, Jackson S, McAlpine A. The advanced clinical nurse a new model of practise: meeting the challenge of peak activity periods. Aust Emerg Nurs J 1999;2:26-8.

6. Cashin A, Connell J, Christofis L, Lentakis A, Rossi M, Crellin D. Clinical initiative nurses and nurse practitioners in the emergency department: what's in a name? Aust Emerg Nurs $J$ 2007;10:73-9.

7. Henderson A. Emotional labor and nursing: an underappreciated aspect of caring work. Nurs Inq 2001;8:130-8.

8. Von Dietze E, Orb A. Compassionate care: a moral dimension of nursing. Nurs Inq 2000;7:166-74.

9. Fry M, Ruperto K, Jarrett K, Wheeler J, Fong J, Fetchet W. Managing the wait: clinical initiative nurses' perceptions of an extended practice role. Aust Emerg Nurs J 2013;15(4):202-10.

10. St George Hospital. Emergency department first net data. Sydney: South Eastern Sydney Illawarra Health Services; 2010.

11. Wollongong Hospital W. Emergency department data manager: Emergency department firstnet data report. New South Wales: South East Sydney Illawarra Health Services; 2012.

12. Gibbs G. Analyzing qualitative data. London: Sage Press; 2007.

13. Lichtman M. Qualitative research in education: a users guide. 2nd ed. Thousand Oaks, CA: Sage; 2010.

14. Brodie P, Irving K. Assessment in work-based learning: investigating a pedagogical approach to enhance student learning. Assess Eval Higher Educ 2007;32:11-9.

15. Davidson N, Williams K. Compassion in nursing 2: factors that influence compassionate care in clinical practice. Nurs Times 2009;105:37.

16. Caris-Verhallen W, Kerkstra A, Bensing J. Non-verbal behaviour in nurse-elderly patient communication. $J$ Adv Nurs 1999;29:808-18.

17. Staden H. Alertness to the needs of others: a study of the emotional labour of caring. J Adv Nurs 1998;27:147-56.

18. Phillips $S$. Labouring the emotions: expanding the remit of nursing work? J Adv Nurs 1996;24:139-43.

19. Downey G. Practice without theory: the imitation bottleneck in embodied knowledge. J Roy Anthropol Inst 2010;16:S22-40.

20. Sawbridge $Y$, Hewison A. Time to care? Responding to concerns about poor nursing care. Policy Paper 12. Birmingham: Health Services Management Centre, University of Birmingham; 2011.

21. Baggs J, Schmitt M, Mushlin A, et al. Association between nurse-physician collaboration and patient outcomes in three intensive care units. Crit Care Med 1999;27:1991-8.

22. Aitken $L$, Havens $D$, Slone $D$. The magnet nursing services recognition program. Am J Nurs 2000;100:26-36.

23. West M, Dawson J, Admasachew L, Topakas A. NHS staff management and health service quality. Department of Health; 2011. 